\title{
ON THE APPLICATION OF STRUCTURE THEORY TO GROUPS
}

\author{
OYSTEIN ORE
}

The object of the following remarks is to discuss a few of the ideas connected with the application of the theory of structures to group theory. We shall have to suppose that the principal properties of a structure are known. It should only be recalled that a structure is an algebraic system with two dual operations, the union $A \cup B$ and the cross-cut $A \cap B$. The subgroups of a group $G$ form such a structure, and in the following we shall mainly consider structures whose elements are subgroups.

It may be said that the main idea in the application of structure theory to groups lies in the realization of the fact that important parts of the theory of groups are not so much a theory of the properties of the group elements as a theory of subgroups. Hence one can take the point of view that the subgroups rather than the elements may be made the building stones for a theory. It is of course quite interesting to examine to what extent this is possible, but the real usefulness of the idea appears through the various new results to which it leads.

One observes first that there exists a direct analogy between the theory of Dedekind structures and the normal decomposition theory for groups. The analysis of the axiomatic basis for the theorem of Jordan-Hölder was the object of Dedekind's original investigations on "Dualgruppen" or structures. A Dedekind structure is characterized by the relation

$$
A \cap(B \cup C)=B \mathbf{u}(A \cap C),
$$

$A \supset B$,

which holds for any three elements. For such structures the ordinary decomposition theorems like the refinement theorem of SchreierZassenhaus (including the theorem of Jordan-Hölder), the theorem of Schmidt-Remak on direct decompositions, the theorem on irreducible decompositions, and so on, can be derived, and since the Dedekind relation (1) is satisfied for any three normal subgroups, the decomposition theorems for groups must follow.

One of the most interesting points in this theory is the extent to which concepts defined by element properties may be replaced by structural properties. For instance, in all decomposition theorems there occurs isomorphism between components in the various decom- 
positions. Now isomorphism is obviously an element property, but one finds that those isomorphisms which actually occur can be obtained by successive applications of the second law of isomorphism.

$$
A / A \cap B \cong A \text { บ } B / B
$$

This means that these isomorphisms can be replaced by the concept of similarity of quotient structures, since two quotient structures are defined to be similar when one can pass from one to the other through repeated transitions of the form (2).

The theorem of Schmidt-Remak contains the further concept of centrally isomorphic subgroups: Two subgroups are centrally isomorphic when the quotient between corresponding elements lies in the center. This can be replaced again by the structural concept of direct similarity: Two normal subgroups $A$ and $B$ are directly similar when there exists a third $C$ such that

$$
A \cup C=B \cup C,
$$

where $A$ and $C$ and also $B$ and $C$ are relatively prime and hence

$$
A \cap C=B \cap C=E,
$$

where $E$ is the unit group. Conversely the relations (3) and (4) imply that $C$ belongs to the center of $A \cup B$ and $A$ and $B$ are centrally isomorphic.

The Schmidt-Remak theorem has been discussed frequently in the literature, but there are still several questions connected with it which have not yet been solved in a satisfactory manner. The theorem is usually proved in algebra under the assumption of the so-called finite chain condition, that is, the condition that both ascending and descending chains of structure elements should be finite. One knows also, according to an example given by Krull concerning moduli in algebraic rings, that the theorem cannot be true in general when only one chain condition is imposed. For the special case of groups there exist however interesting investigations by Kurosch, Fitting, and Korinek in which the theorem is deduced under very weak conditions. Also for Dedekind structures the theorem can be obtained when only one chain condition holds, but then certain further limitations must be made; for instance it is sufficient to make the rather natural assumption that certain component structures should not be structureisomorphic to proper substructures. These are extensions of the theorem in a different direction from those mentioned for groups, but a common general form is not known. 
Let us return for a moment to the relations (3) and (4). We have already mentioned that they imply that $C$ belongs to the center of $A \cup B$; hence it is an abelian group. Consequently the symmetric relations

$$
\begin{aligned}
& A \cup B=B \cup C=C \cup A=M, \\
& A \cap B=B \cap C=C \cap A=E
\end{aligned}
$$

imply that $A, B, C$, and also $M$ are abelian groups, When this is applied to the three groups $T_{A}, T_{B}, T_{C}$ obtained from

$$
T_{A}=(A \cap(B \cup C)) \cup(B \cap C)
$$

by permutations, it yields the rather interesting fact that for any three normal subgroups $A, B, C$ the quotient group

$$
(A \cup B) \cap(B \cup C) \cap(C \cup A) /(A \cap B) \cup(B \cap C) \text { บ }(C \cap A)
$$

is abelian. It is not an arbitrary abelian group since its invariants must occur in pairs, but any abelian group with this property is so representable.

Up to the present we have discussed the decomposition theorems for normal subgroups. Now one can also examine the possibility of extending some of these results to certain non-normal subgroups. The first difficulty one encounters in such an investigation is the fact that the quotient groups are only defined for normal subgroups. In a structure one has the simpler situation that there is a quotient structure $A / B$ associated with every pair of elements $A \supset B$. This suggests the introduction also in groups of an algebraic system $A / B$ defined for all subgroups $A \supset B$. The system $A / B$ consists of the cosets $a \cdot B$ (or $B \cdot a$ ) as in the normal case. The difference lies in the fact that when the elements of two cosets are multiplied, they will usually not belong to a single third coset but will distribute themselves over a number of cosets

$$
a_{1} B \cdot a_{2} B=\left\{\cdots, a_{i} B, \cdots\right\} .
$$

This leads us to define the algebraic system $A / B$ as a multigroup, that is, an algebraic system with a many-valued product operation. In general a multigroup satisfies the two axioms:

(i) The associative law holds.

(ii) The linear relations

$$
a x \supset b, \quad y a \supset b
$$

shall always be solvable, that is, there shall exist an $x$ such that b occurs among the various values of the product ax. 
The theory of such multigroups or hypergroups has first been studied by Marty and Wall. In a recent paper by Dresher and Ore the decomposition theory has been investigated on the basis of the theory of Dedekind structures.

The theory presents considerable complications in comparison with ordinary group theory. Unit elements such that

$$
a e \supset a, \quad e a \supset a
$$

for all $a$ may or may not exist. If they exist, right or left inverses can be defined but are not uniquely determined. Most investigations involve closed sub-multigroups, where we define a sub-multigroup $A$ to be closed when it has the property that if $a$ and $b$ belong to $A$ then all $x$ and $y$ satisfying (6) also belong to A. Normality may be defined in two ways: The condition

$$
g A=A g
$$

for all $g$ is sufficient to derive the Dedekind relation and a theorem of Jordan-Hölder for closed normal sub-multigroups. The stronger condition

$$
g A g^{-1} \subset A
$$

for some inverse $g^{-1}$ implies that the quotient multigroup defined by $A$ is an ordinary group. There exists also an interesting type of multigroups with composition chains in which every quotient multigroup is a group.

Let us now return to the extension of the decomposition theorems in ordinary groups. One finds easily that the Dedekind relation (1) also holds for all $A$ if $B$ and $C$ are permutable groups, that is, there exist relations

$$
b_{1} \cdot c_{1}=c_{2} \cdot b_{2}
$$

for their elements. From this fact follows the weak form for the theorem of Jordan-Hölder that two chains of subgroups which satisfy certain permutability conditions must always have the same length and their indices must be the same in some order. A similar result holds for irreducible decompositions. The closest analogy to the normal case is obtained for the so-called quasi-normal subgroups. Normality of a subgroup $A$ is defined by the relation

$$
g \cdot a=a^{\prime} \cdot g
$$

for all $g$. In this permutability relation one may replace the absolute 
invariance of $g$ by structural invariance; that is, $g$ shall remain in the same cyclic subgroup, hence we shall have the relation

$$
g \cdot a=a^{\prime} \cdot g^{n},
$$

where the exponent $n$ may depend upon $g$ and $a$. Subgroups $A$ satisfying this condition shall be called quasi-normal. For quasi-normal subgroups the analog to the theorem of Jordan-Hölder holds with the only difference that isomorphism of the quotient groups is replaced by structure isomorphism of the quotient multigroups. Also for the decompositions into irreducible quasi-normal components the results are analogous to the normal case.

One of the interesting applications of the ideas of structure theory to groups is to be found in the principle of duality. The formulation of the structure axioms shows that they are dual in character in the sense that they remain the same when union and cross-cut are interchanged. This implies that every structure theorem has a dual counterpart which one obtains by such an interchange, and consequently those group theorems which are purely structural in nature can be dualized. But obviously in most cases the group theorems depend on the special character of the group as an algebraic system. On the other hand to each theorem containing only subgroups and their cross-cut and union a dual theorem can be formulated. The fundamental question is then when this dual theorem is a true theorem or under what conditions it is valid. We shall not discuss this any further, but it should be noted that various important problems in group theory may be considered to be of this nature.

The Dedekind relation is self-dual, and the ordinary normal decomposition theorems are structural in nature and hence may be dualized directly. The self-dual theorems are usually of particular interest. Both the refinement theorem of Schreier-Zassenhaus for principal series, including the Jordan-Hölder case, as well as the theorem of Schmidt-Remak on direct decompositions, have this property. Another pretty self-dual theorem is the one we have already mentioned stating that the quotient group (5) is abelian.

To illustrate the process of dualization let us consider only one example. A group $G$ may be generated in various ways by means of elements

$$
G=\left\{g_{1}, g_{2}, \cdots\right\} .
$$

All those elements $f_{1}, f_{2}, \cdots$ which are superfluous in any such representation form a group, the $\phi$-group of $G$. To dualize this concept we shall have to define it in a slightly different, but purely structural 
form. We consider all representations of $G$ as the union of subgroups

$$
G=A_{1} \mathbf{u} A_{2} \mathbf{u} \cdots \text {. }
$$

All the subgroups $F_{1}, F_{2}, \cdots$ which are superfluous in any such representation form a structure, and their union is the $\phi$-group of $G$. One also derives the theorem that the $\phi$-group is the cross-cut of all the maximal subgroups of $G$. The dual of the representation of $G$ as the union of subgroups is the representation of the unit element as the cross-cut of subgroups

$$
E=A_{1} \cap A_{2} \cap \cdots .
$$

Those subgroups which can be omitted in any such representation again form a structure, their union $\phi^{*}$ is a characteristic subgroup and the quotient group $G / \phi^{*}$ is the dual of the $\phi$-group. One sees that $\phi^{*}$ is the union of all minimal subgroups of $G$; hence it is the subgroup generated by all elements of prime order when we suppose that all elements have finite orders. This dualization process also raises an interesting problem. The structure of the $\phi$-group is particularly simple since it is known to be the direct product of its Sylow groups. The dual problem is to determine whether one can in general say anything about the structure of the group $G / \phi^{*}$, namely the quotient group of a group with respect to the subgroup generated by the elements of prime order.

Finally one might mention the problem of the structural relations. In the theory of structures certain relations like the Dedekind relation or the distributive relation

$$
A \cap(B \cup C)=(A \cap B) \cup(A \cap C)
$$

are of fundamental importance. Also for groups it is of interest to know when such relations hold for the subgroups, and it is of particular interest to know which groups are characterized by the fact that a certain structural relation holds for all subgroups. A few results in this direction have been obtained, but they will not be discussed here.

\section{REFERENCES}

Oystein Ore, On the foundation of abstract algebra. I, Annals of Mathematics, vol. 36 (1935), pp. 406-437; II, ibid., vol. 37 (1936), pp. 265-292.

-, Structures and group theory. I, Duke Mathematical Journal, vol. 3 (1937), pp. 149-174; II, ibid., vol. 4 (1938), pp. 247-269.

Dresher and Ore, Theory of multigroups, American Journal of Mathematics, vol. 60 (1938), pp. 705-733.

\section{YALE UNIVERSITY}

